\title{
Revalidation of Apostolepis barrioi (Serpentes: Dipsadidae)
}

\author{
Hugo Cabral, ${ }^{1,2}$ Thales de Lema, ${ }^{3}$ and Márcia Ferret Renner ${ }^{3}$ \\ ${ }^{1}$ Asociación Guyra Paraguay, Avenida Coronel Carlos Bóveda, Parque Asunción Verde, Viñas Cué, Paraguay. \\ E-mail: huguitocabral@gmail.com. \\ ${ }^{2}$ Instituto de Investigación Biológica del Paraguay, Del Escudo 1607, Asunción, Paraguay. \\ ${ }^{3}$ Pontifícia Universidade Católica do Rio Grande do Sul, Museu de Ciências e Tecnologia, Laboratório de Sistemática de \\ Vertebrados, Avenida Ipiranga 6681, bld. 40, Caixa Postal 1429, 90619-900, Porto Alegre, RS, Brazil.
}

\begin{abstract}
Revalidation of Apostolepis barrioi (Serpentes: Dipsadidae). With 32 species, Apostolepis is the most diverse genus in the tribe Elapomorphini. The description of Apostolepis barrioi is based on one specimen and the taxon later was synonymized with $A$. dimidiata. Based on a examination of new specimens, the taxon is resurrected to full species rank. Apostolepis barrioi differs from all congeners by having an immaculate white venter, narrow dorsolateral stripes not in contact with the ventrals, and a terminal black shield. With the addition of A. barrioi, the number of species of the genus increases to 33; however, more systematic research on the status of the species in the group is required, especially for those species that were described on the basis of only on few specimens.
\end{abstract}

Keywords: Atlantic Forest, Cerrado, Brazil, Paraguay, South America.

\begin{abstract}
Resumo
Revalidação de Apostolepis barrioi (Serpentes: Dipsadidae). Apostolepis é o gênero mais diversificado da tribo Elapomorphini, com 32 espécies reconhecidas. Apostolepis barrioi foi descrita com base em um único exemplar e então sinonimizada a $A$. dimiata. Depois de uma reavaliação de novos dados, reconhecemos Apostolepis barrioi como um táxon válido que se distingue das demais espécies do gênero por apresentar o ventre e as escamas gulares de cor branca imaculada, faixas dorsais mais finas que podem ou não apresentar contato com as escamas ventrais e placa terminal negra. Com a revalidação de Apostolepis barrioi o número de espécies do gênero aumenta para 33. No entanto, mais estudos taxonômicos relacionados aos grupos do gênero são necessários, especialmente com aquelas espécies cujas descrições estão baseadas em poucos exemplares.
\end{abstract}

Palavras-chave: América do Sul, Brasil, Cerrado, Mata Atlântica, Paraguai. 


\section{Introduction}

Apostolepis Cope, 1862 is a genus of fossorial snakes that is widely distributed in South America east of the Andes, from Guyana to northern Argentina (Giraudo and Scrocchi 1998, Lema 2001, Nogueira et al. 2012). The genus was placed in the tribe Elapomorphini along with Phalotris Cope, 1862, Elapomorphus Wiegmann, 1843, and Coronelaps Lema and Hofstadler-Deiques, 2010 (Zaher 1999, Zaher et al. 2009, Lema and Hofstadler-Deiques 2010, Grazziotin et al. 2012, Pyron et al. 2013). Currently, 32 species of Apostolepis are known (Uetz et al. 2017); thus, it is the most diverse genus of the tribe.

The description of Apostolepis barrioi Lema, 1978 was based on a single specimen from Paraguay (Lema 1978) (Figure 1), which was differentiated from A. dimidiata (Jan, 1862) mainly by the absence of ventral blotches in $A$. barrioi, in contrast to the ventral dark blotches in A. dimidiata. Based on an analysis of color variation in A. dimidiata, Lema (1993) reported that the species is polymorphic and described the following morphs: (1) melanistic, with black dorsolateral stripes and belly; (2) two rows of black ventral blotches, forming a narrow white midventral area; and (3) albinistic, with black dorsolateral stripes not reaching the ventrals, and an immaculate belly. Based primarily on meristic data and their sympatry, Lema (1993) placed $A$. barrioi and A. villaricae (Lema, 1978) in the synonymy of $A$. dimidiata.

Recently, the "Para la Tierra" NGO from Paraguay published photographs of a living snake (Figure 1) from Laguna Blanca $\left(23^{\circ} 47^{\prime} 56^{\prime \prime S}\right.$, $\left.56^{\circ} 17^{\prime} 32^{\prime \prime} \mathrm{W}\right)$ located in the Departamento of San Pedro, in the center of the Oriental Region of Paraguay, and deposited in the Museo Nacional de Historia Natural del Paraguay (MNHNP). Herein, we report the results of meristic and morphological analyses of this individual snake from Paraguay and numerous additional specimens, thereby providing support for the revalidation $A$. barrioi.

\section{Materials and Methods}

Data were gathered for Apostolepis dimidiata from specimens and databases in many collections. Photographs of living individuals were provided by colleagues (Acknowledgments). Collections in the following institutions [indicated by codes used by Sabaj-Pérez (2016)] were used: USA: AMNH, MCZ, USNM; United Kingdom: BMNH; Brazil: MZUSP, IBSP, MCN, MHNCI, MNRJ, UFU; Paraguay: MNHNP; Argentina: MACN, MLP, UNNEC, CHUNAM; Austria: NMW; Russia: ZISP; Germany: ZMB, ZSM. See Appendix I for specimens examined and localities.

Dowling's (1951) conventions for ventral scale counts were followed. Snout-vent and tail lengths were measured with a tape measure to the nearest millimeter. Other measurements were taken with a dial caliper $(0.01 \mathrm{~mm})$. Head pholidosis includes rostral, nasal, frontal and prefrontal, parietals, supralabials and infralabials, preoculars, postoculars. For supralabials and infralabials, the parenthetical range represents scales in contact with the orbit and chinshields, respectively. The nuchal collar is posterior to the head coloration; in some cases, the nuchal collar coloration is continuous with that of the head. Description of coloration is based on preserved specimens. Distribution maps were created with ArcGis (v10.1, ESRI). Ecoregions are those of Dinerstein et al. (1995), and we followed the group definitions of Nogueira et al. (2012). When present, the coloration of nuchal, cervical, and post-cervical collars were compared the information in the original descriptions of the species. Any stripe in the vertebral region is referred to as a "vertebral stripe." Dorsolateral stripes usually are black and several scales in width; they differ from the much narrower stripes present in some species of Apostolepis.

\section{Results}

On reviewing Lema $(1978,1993)$, we noticed that the albinistic morph was found mainly in 

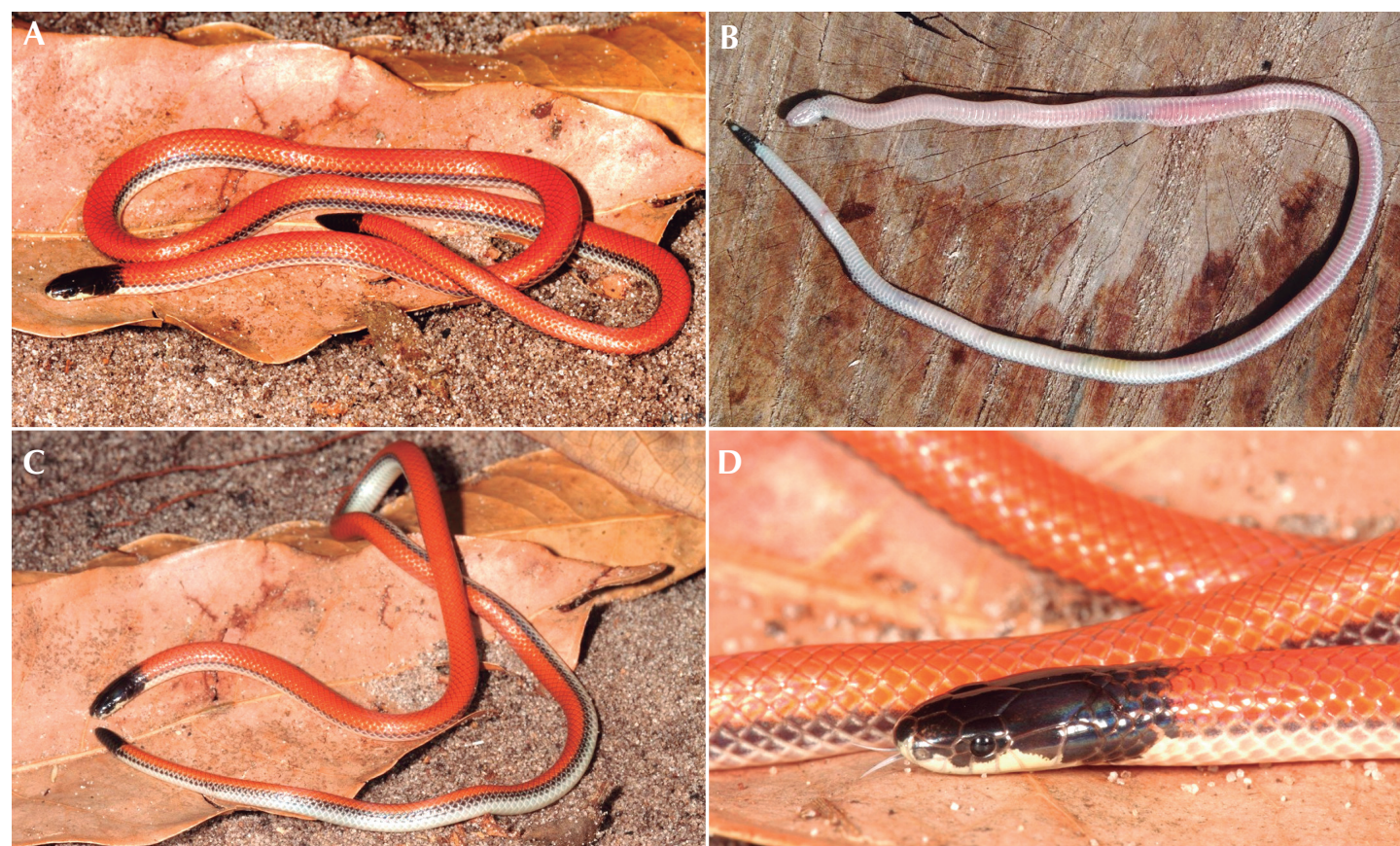

Figure 1. Apostolepis barrioi from Laguna Blanca, Paraguay. (A) Dorsal view; (B) ventral view, showing the absence of black coloration on ventral scales; $(\mathbf{C})$ dorsal view showing the black dorsolateral stripes not reaching the ventral scales; (D) details of the head.

Paraguay, and when we compared data from a large sample $(N=142)$ of $A$. dimidiata, we noted some differences between the albinistic and the other morphs. The analysis included three morphs: melanistic, belly with a narrow white midventral area, and albinistic. The albinistic form is occurs in Paraguay and Brazil (Figure 2) and is characterized by the immaculate white venter, and dorsolateral black stripes that do not reach the ventral scales (Figure 3). The other two morphs occur in Argentina, Brazil, and Paraguay and have completely dark venters or dark coloration on the edges of the ventral scales, lateral dark stripes that always reach the ventral scales, and an incomplete vertebral stripe (Giraudo 2002). In A. barrioi, ventrals range from 222-256 and subcaudals from 23-35; in A. dimidiata, ventrals range from 214-264 and subcaudals from 22-39. The Table 1 summarizes the measurements and range of the pholidosis of the specimens examined. The numbers of ventrals and subcaudals in the two species are the same. Based on the presence or absence of dark pigments on the belly, we distinguished only two morphs among the three described: (1) individuals with completely white bellies; and (2) snakes with either entirely black bellies, or black on the edges of the ventrals and lowest dorsal scales. These two morphs seem to be different species because these characters seem to be conserved. The holotype of Apostolepis barrioi matches the characteristics of the albinistic morph. Below, the holotype of $A$. barrioi is redescribed and the species revalidated. Data describing morphological variation of $A$. barrioi are presented. Apostolepis barrioi belongs to the A. dimidiata Group, which is characterized by the presence of a pointed rostral, elongate 


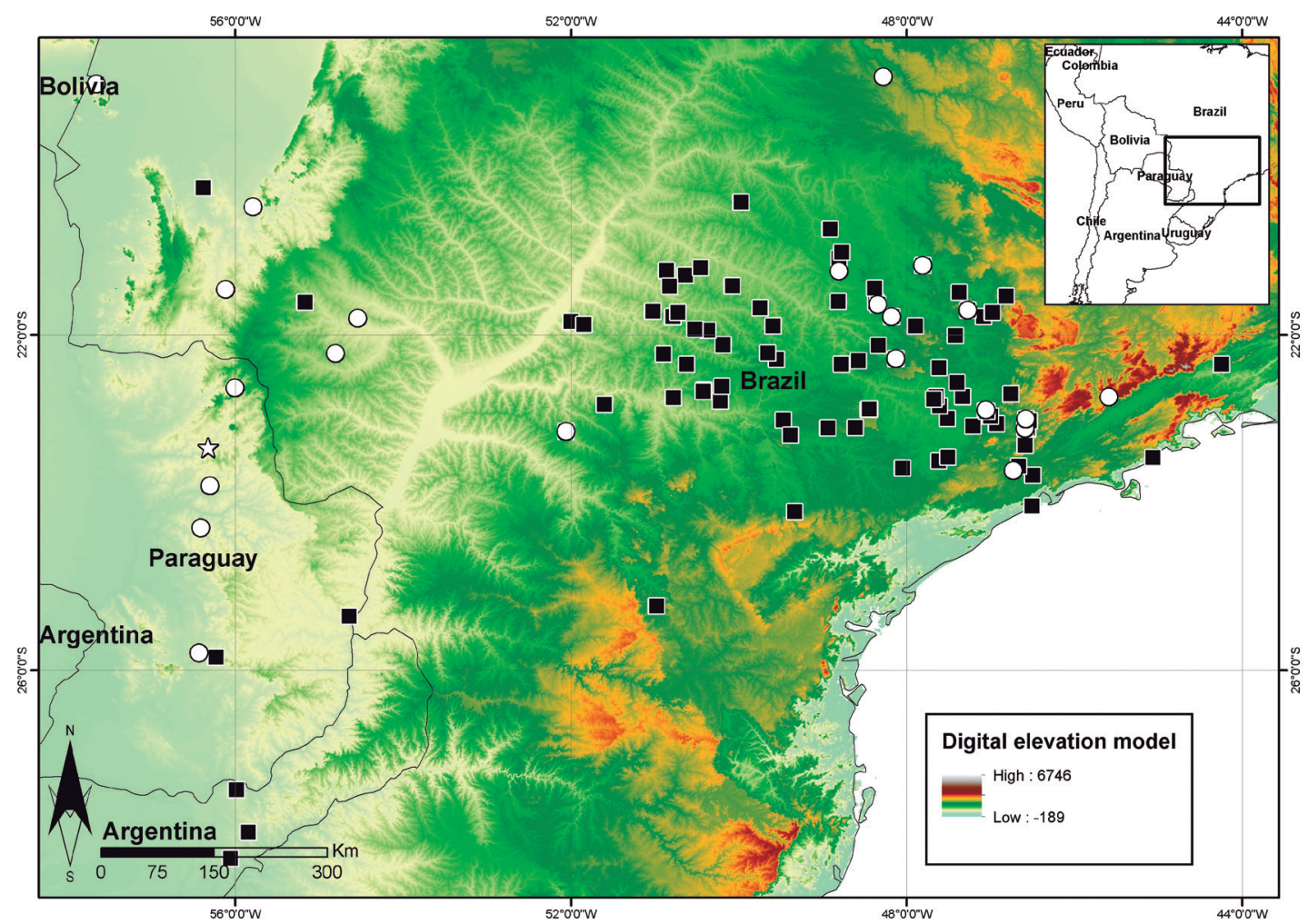

Figure 2. Distribution of Apostolepis barrioi and $A$. dimidiata. Black squares represent the records of $A$. dimidiata, white circles the records of $A$. barrioi, and white star represents the type locality of $A$. barrioi.

white supralabial blotch, rounded terminal shield, and a body with black dorsolateral stripes (Nogueira et al. 2012).

Apostolepis barrioi Lema, 1978 Apostolepis dimidiata Lema, 1993

Holotype.-MACN 49402 (CHINM 3309 in the original description, young male); col: G. J. Williner, 1965 February; from Avelino Barrio Collection, Buenos Aires, Argentina (Figure 4).

Type locality.-Cororó, Río Ypané, Departamento Concepción, Paraguay (Figure 2). The type locality is an area of transition from the Atlantic Forest to the Cerrado and Chaco Ecorregión (Dinerstein et al. 1995).
Table 1. Scale counts of ventrals (Vent), subcaudals (SC), and measurements of snout-vent length $(\mathrm{SVL})$, tail length (TL) and ratio between $\mathrm{TL}$ and SVL of Apostolepis barrioi and $A$. dimidiata. The number of specimens examined $(N)$ is given in parentheses.

\section{A. barrioi}

$222-256(34)$

23-55 (34)

$188-542(17)$

SVL

TL

Ratio

SC

16-45 (18)

$0.06-0.11(17)$

\section{A. dimidiata}

214-264 (113)
16-60 (112)

22-39 (111)

180-676 (112)

$0.05-0.12(112)$ 


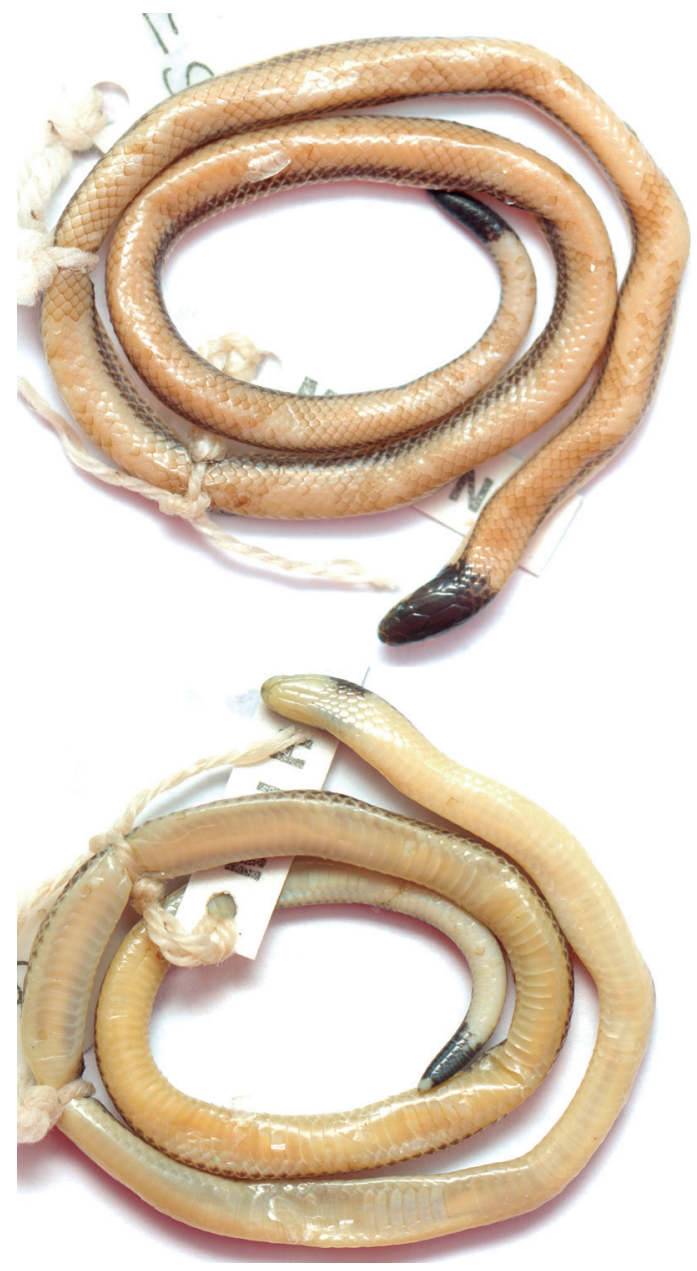

Figure 3. Dorsal and ventral view of Apostolepis barrioi (MNHNP 11548) showing the immaculate white ventral coloration.

Distribution.-From Minas Gerais and São Paulo to Mato Grosso do Sul states, Brazil, to northern and central Paraguay (Figure 2).

Diagnosis.-Apostolepis barrioi differs from its congeners by the following characteristics: absence of white nuchal collar; presence of a complete black nuchal collar; venter immaculate white; prefrontal uniformly black; gular region white; narrow dorsolateral stripes

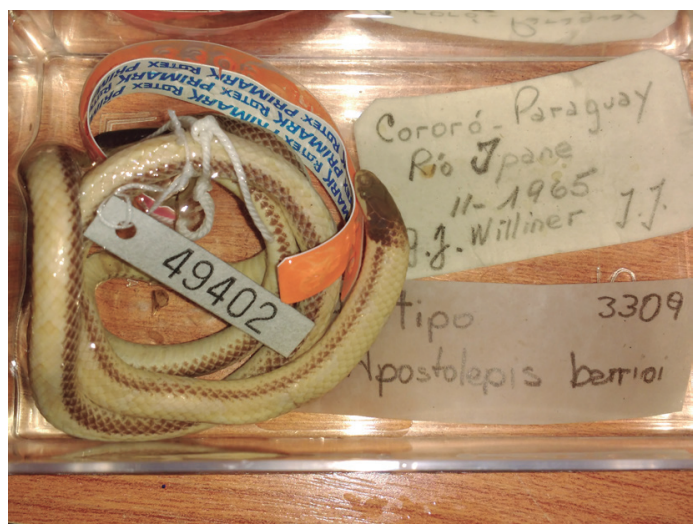

Figure 4. Holotype of Apostolepis barrioi (MACN 49402).

not reaching the ventrals; terminal shield black. In a complete diagnosis, A. barrioi is compared with all congeners; characters in parentheses are those of A. barrioi. Table 2 summarizes these differences.

Apostolepis barrioi is distinguished from A. albicollaris, A. ammodites, A. arenaria, A. assimilis, A. borelli, A. cearensis, A. cerradoensis, A. dorbgnyi, A. flavotorquata, A. gaboi, A. multicincta, A. nelsonjorgei, A. nigroterminata, A. phillipsi, A. quirogai, A. tenuis, and A. tertulianobeui by the white or yellow nuchal collars in these species (black nuchal collar in A. barrioi). Apostolepis christinae, A. goiasensis, A. longicaudata, A. niceforoi, A. nigrolineata, A. pymi, and $A$. serrana lack nuchal collars (present in A. barrioi). Apostolepis albicollaris, A. ammodites, A. arenaria, A. assimilis, A. borelli, A. cearensis, A. cerradoensis, A. dorbgnyi, A. flavotorquata, A. gaboi, A. multicincta, A. nigroterminata, A. phillipsi, A. quirogai, and A. tertulianobeui have black cervical collars (cervical collar absent in A. barrioi). Apostolepis ammodites has a white postcervical collar (postcervical absent in A. barrioi). Apostolepis arenaria, A. borelli, A. breviceps, A. cerradoensis, A. christinae, A. dimidiata, A. gaboi, A. goiasensis, A. intermedia, A. lineata, A. longicaudata, A. nelsonjorgei, A. niceforoi, A. nigrolineata, A. nigroterminata, A. phillipsi, A. 
Table 2. Selected external characters for Apostolepis species. Data extracted from original descriptions and taxonomic comparisons (Duméril et al. 1854, Cope 1887, Peracca 1904, Amaral 1921, 1935, Ruthven 1927, Peters and Orejas-Miranda 1972, Lema 1978, 1998, 2002, 2003, 2004a,b, 2016, Rodrigues 1993, Giraudo and Scrocchi 1998, Harvey 1999, Harvey et al. 2001, Lema and Renner 2004, 2005, 2012, Ferrarezi et al. 2005, Lema and Albuquerque 2010, Curcio et al. 2011, Albuquerque and Lema 2012, Loebmann and Lema 2012, Nogueira et al. 2012, Entiauspe-Neto and Lema 2015).

\begin{tabular}{|c|c|c|c|c|c|c|c|c|}
\hline \multirow{2}{*}{ Species } & \multicolumn{8}{|c|}{ Characters } \\
\hline & $\begin{array}{l}\text { Nuchal } \\
\text { collar }\end{array}$ & $\begin{array}{c}\text { Cervical } \\
\text { collar }\end{array}$ & $\begin{array}{l}\text { Postcervi- } \\
\text { cal collar }\end{array}$ & $\begin{array}{l}\text { Vertebral } \\
\text { stripe }\end{array}$ & $\begin{array}{l}\text { Body } \\
\text { stripes }\end{array}$ & Body lines & $\begin{array}{l}\text { Tip of } \\
\text { the tail }\end{array}$ & $\begin{array}{c}\text { Ventral } \\
\text { coloration }\end{array}$ \\
\hline $\begin{array}{l}\text { Apostolepis } \\
\text { albicollaris Lema, } \\
2002\end{array}$ & White & Black & Absent & Absent & $\begin{array}{c}\text { Dorsolat- } \\
\text { eral }\end{array}$ & Absent & $\begin{array}{c}\text { Black } \\
\text { dorso- } \\
\text { ventrally }\end{array}$ & $\begin{array}{l}\text { Ventral surface } \\
\text { with a pair of } \\
\text { large ventrolateral } \\
\text { black spots on } \\
\text { each ventral } \\
\text { shield }\end{array}$ \\
\hline $\begin{array}{l}\text { Apostolepis } \\
\text { ambiniger (Peters, } \\
1869 \text { ) }\end{array}$ & Black & Absent & Absent & Absent & Absent & Absent & $\begin{array}{c}\text { White } \\
\text { ventrally }\end{array}$ & Uniform white \\
\hline $\begin{array}{l}\text { Apostolepis } \\
\text { ammodites } \\
\text { Ferrarezi, Erritto } \\
\text { Barbo and } \\
\text { Albuquerque, } \\
2005\end{array}$ & White & Black & White & Absent & Absent & Absent & Black & Uniform white \\
\hline $\begin{array}{l}\text { Apostolepis } \\
\text { arenaria } \\
\text { Rodrigues, } 1993\end{array}$ & White & Black & Absent & Present & Present & Five & Black & Uniform white \\
\hline $\begin{array}{l}\text { Apostolepis } \\
\text { assimilis (Reinhart, } \\
\text { 1861) }\end{array}$ & White & Black & Absent & Absent & Absent & Absent & Black & Uniform white \\
\hline $\begin{array}{l}\text { Apostolepis barrioi } \\
\text { Lema, } 1978\end{array}$ & Black & Absent & Absent & Absent & Present & Absent & White & Uniform white \\
\hline $\begin{array}{l}\text { Apostolepis } \\
\text { borellii Peracca, } \\
1904\end{array}$ & White & Black & Absent & Present & Present & Four & White & Uniform white \\
\hline $\begin{array}{l}\text { Apostolepis } \\
\text { breviceps Harvey, } \\
\text { Gonzalez and } \\
\text { Scrocchi, } 2001\end{array}$ & Black & Absent & Absent & $\begin{array}{c}\text { Faint } \\
\text { vertebral } \\
\text { stripe, only } \\
\text { on anterior } \\
\text { half of body }\end{array}$ & Absent & Absent & Creamy & Uniform white \\
\hline $\begin{array}{l}\text { Apostolepis } \\
\text { cearensis Gomes, } \\
1915\end{array}$ & White & Black & Absent & Absent & Absent & Absent & Black & Uniform white \\
\hline $\begin{array}{l}\text { Apostolepis } \\
\text { cerradoensis } \\
\text { Lema, } 2003\end{array}$ & White & Black & Absent & Vestigial & $\begin{array}{c}\text { Dorsolat- } \\
\text { eral }\end{array}$ & Absent & White & Uniform white \\
\hline
\end{tabular}


Table 2. Continued.

\begin{tabular}{|c|c|c|c|c|c|c|c|c|}
\hline \multirow{2}{*}{ Species } & \multicolumn{8}{|c|}{ Characters } \\
\hline & $\begin{array}{l}\text { Nuchal } \\
\text { collar }\end{array}$ & $\begin{array}{l}\text { Cervical } \\
\text { collar }\end{array}$ & $\begin{array}{l}\text { Postcervi- } \\
\text { cal collar }\end{array}$ & $\begin{array}{l}\text { Vertebral } \\
\text { stripe }\end{array}$ & Body stripes & $\begin{array}{l}\text { Body } \\
\text { lines }\end{array}$ & $\begin{array}{l}\text { Tip of } \\
\text { the tail }\end{array}$ & Ventral coloration \\
\hline $\begin{array}{l}\text { Apostolepis } \\
\text { christineae Lema, } \\
2002\end{array}$ & Absent & Absent & Absent & Present & Absent & Five & White & Uniform white \\
\hline $\begin{array}{l}\text { Apostolepis } \\
\text { dimidiata (Jan, } \\
1862 \text { ) }\end{array}$ & Black & Absent & Absent & $\begin{array}{l}\text { Vestigial or } \\
\text { absent }\end{array}$ & $\begin{array}{l}\text { Dorsolat- } \\
\text { eral }\end{array}$ & Absent & White & $\begin{array}{c}\text { Heavily } \\
\text { pigmented with } \\
\text { black, only the } \\
\text { edge of ventrals } \\
\text { white }\end{array}$ \\
\hline $\begin{array}{l}\text { Apostolepis } \\
\text { dorbignyi } \\
\text { (Schlegel, 1837) }\end{array}$ & White & Black & Absent & Absent & Absent & Absent & Cream & Uniform white \\
\hline $\begin{array}{l}\text { Apostolepis } \\
\text { flavotorquata } \\
\text { (Duméril, Bibron } \\
\text { and Duméril, } \\
\text { 1854) }\end{array}$ & Yellow & Black & Absent & Absent & Absent & Absent & Cream & Uniform white \\
\hline $\begin{array}{l}\text { Apostolepis gaboi } \\
\text { Rodrigues, } 1993\end{array}$ & Yellow & Black & Absent & Present & Present & Seven & Black & Uniform white \\
\hline $\begin{array}{l}\text { Apostolepis } \\
\text { goiasensis Prado, } \\
1942\end{array}$ & Absent & Absent & Absent & Present & Absent & Three & White & Uniform white \\
\hline $\begin{array}{l}\text { Apostolepis } \\
\text { intermedia } \\
\text { Koslowsky, } 1898\end{array}$ & Black & Absent & Absent & Present & Absent & Five & White & Uniform white \\
\hline $\begin{array}{l}\text { Apostolepis lineata } \\
\text { Cope, } 1887\end{array}$ & Black & Absent & Absent & Present & Present & Five & White & Uniform white \\
\hline $\begin{array}{l}\text { Apostolepis } \\
\text { longicaudata } \\
\text { Gomes, } 1921\end{array}$ & Absent & Absent & Absent & Present & Absent & Five & White & Uniform white \\
\hline $\begin{array}{l}\text { Apostolepis } \\
\text { multicincta } \\
\text { Harvey, } 1999\end{array}$ & White & Black & Absent & Absent & Absent & Absent & $\begin{array}{l}\text { Mostly } \\
\text { gray }\end{array}$ & Uniform white \\
\hline $\begin{array}{l}\text { Apostolepis } \\
\text { nelsonjorgei Lema } \\
\text { and Renner, } 2004\end{array}$ & White & Absent & Absent & Present & Present & Five & Black & Uniform white \\
\hline $\begin{array}{l}\text { Apostolepis } \\
\text { niceforoi Amaral, } \\
1935\end{array}$ & Absent & Absent & Absent & Present & Present & Seven & Black & Uniform white \\
\hline $\begin{array}{l}\text { Apostolepis } \\
\text { nigrolineata } \\
\text { (Peters, 1869) }\end{array}$ & Absent & Absent & Absent & Present & Present & $\begin{array}{c}5 \text { in } \\
\text { juveniles, } \\
3 \text { in adults }\end{array}$ & White & Uniform white \\
\hline
\end{tabular}


Table 2. Continued.

\begin{tabular}{|c|c|c|c|c|c|c|c|c|}
\hline \multirow{2}{*}{ Species } & \multicolumn{8}{|c|}{ Characters } \\
\hline & $\begin{array}{l}\text { Nuchal } \\
\text { collar }\end{array}$ & $\begin{array}{l}\text { Cervical } \\
\text { collar }\end{array}$ & $\begin{array}{l}\text { Postcervi- } \\
\text { cal collar }\end{array}$ & $\begin{array}{l}\text { Vertebral } \\
\text { stripe }\end{array}$ & Body stripes & $\begin{array}{l}\text { Body } \\
\text { lines }\end{array}$ & $\begin{array}{l}\text { Tip of } \\
\text { the tail }\end{array}$ & Ventral coloration \\
\hline $\begin{array}{l}\text { Apostolepis } \\
\text { nigroterminata } \\
\text { Boulenger, } 1896\end{array}$ & Cream & $\begin{array}{l}\text { Irregular } \\
\text { brown } \\
\text { collar }\end{array}$ & Absent & Present & Present & Five & Cream & Uniform white \\
\hline $\begin{array}{l}\text { Apostolepis } \\
\text { phillipsi Harvey, } \\
1999\end{array}$ & White & $\begin{array}{l}\text { Irregular } \\
\text { black } \\
\text { collar }\end{array}$ & Absent & Present & Present & Five & White & Uniform white \\
\hline $\begin{array}{l}\text { Apostolepis } \\
\text { polylepis Amaral, } \\
1921\end{array}$ & Black & Absent & Absent & Present & Present & Absent & Black & Uniform white \\
\hline $\begin{array}{l}\text { Apostolepis pymi } \\
\text { Boulenger, } 1903\end{array}$ & Absent & Absent & Absent & Present & Present & Three & Black & Uniform white \\
\hline $\begin{array}{l}\text { Apostolepis } \\
\text { quirogai Giraudo } \\
\text { and Scrocchi, } \\
1998\end{array}$ & White & Black & Absent & $\begin{array}{l}\text { Reduced to } \\
\text { small dots }\end{array}$ & $\begin{array}{l}\text { Dorsolat- } \\
\text { eral }\end{array}$ & Absent & $\begin{array}{l}\text { Center } \\
\text { lighter }\end{array}$ & $\begin{array}{l}\text { Pigmented with } \\
\text { black }\end{array}$ \\
\hline $\begin{array}{l}\text { Apostolepis } \\
\text { serrana Lema and } \\
\text { Renner, } 2006\end{array}$ & Absent & Absent & Absent & - & - & - & White & - \\
\hline $\begin{array}{l}\text { Apostolepis striata } \\
\text { Lema, } 2004\end{array}$ & Black & Absent & Absent & Present & Present & Five & White & Uniform white \\
\hline $\begin{array}{l}\text { Apostolepis tenuis } \\
\text { Ruthven, } 1927\end{array}$ & White & Absent & Absent & Present & Present & Absent & White & Uniform white \\
\hline $\begin{array}{l}\text { Apostolepis } \\
\text { tertulianobeui } \\
\text { Lema, } 2004\end{array}$ & White & Black & Absent & Absent & Absent & Absent & White & Uniform white \\
\hline $\begin{array}{l}\text { Apostolepis vittata } \\
\text { (Cope, 1887) }\end{array}$ & Black & Absent & Absent & Present & Present & Five & White & Uniform white \\
\hline
\end{tabular}

polylepis, A. pymi, A. quirogai, A. striata, A. tenuis, and $A$. vittata have a vertebral stripe, which sometimes is reduced to small dots (absence of vertebral stripe or dots in A. barrioi). Apostolepis arenaria, A. borelli, A. christinae, A. gaboi, A. goiasensis, A. intermedia, A. lineata, A. longicaudata, A. nelsonjorgei, A. niceforoi, A. nigrolineata, A. nigroterminata, A. phillipsi, A. pymi, A. striata, and A. vittata have five or seven narrow black dorsal stripes (presence of two broad black dorsolateral stripes in A. barrioi). In A. albicollaris, A. ammodites, A. arenaria, A. assimilis, A. cearensis, A. gaboi, A. nelsonjorgei, A. niceforoi, A. polylepis, and A. pymi the tips of the tail are black (tip of the tail white in $A$. barrioi), and in A. albicollaris, A. dimidiate, and A. quirogai, the ventral scales are black (immaculate white ventral scales in A. barrioi). 
Description of the holotype.-Head elongate; snout slender, rounded, and strongly projecting, $1.5 \mathrm{~mm}$ beyond jaws; tongue groove deep; nostril, on anterior part of the nasal, posteriorly oblique. Measurements: head length $11 \mathrm{~mm}$; tail length $37 \mathrm{~mm}$; total length $390 \mathrm{~mm}$; snout-vent length $353 \mathrm{~mm}$; tail length/snout-vent length relation 0.105 , tail long, $10 \%$ of the total length. Dorsal aspect of rostral one-half length of suture between prefrontal; prefrontal shorter than frontal; parietals elongate $(5.5 \times 4.5 \mathrm{~mm})$; nasal large $(2.5 \times 2.0 \mathrm{~mm}$, in contact with prefrontal, and 1st and 2nd supralabials; prefrontal larger than postocular; posterior temporal absent; 5th and 6th supralabials in contact with parietals; ventrals 220; paired subcaudals 27; supralabials 6 (2-3), infralabials 7 (1-4). Mental long and pointed; seven dorsal scale rows on tail. Tail tip rounded, short.

Coloration in preservative.-Head black from the snout posterior to first five dorsal vertebral scales, forming nuchal collar; rostral white or cream, with black blotches medially; supralabials white with black on the suture between postocular and temporals; infralabials white. Two dorsolateral black stripes extending from collar; tail with dark blotches on 11 dorsal scales.

Coloration in life.-Head entirely black in dorsal view with white on only some supralabials and rostrum. Ventral aspect of infralabials and chinshields completely white. Black coloration extends onto the first three dorsal scales; two dorsolateral stripes, two dorsal scales wide, extending from black collar posteriorly along flanks and onto the tail. Flanks below dorsolateral black stripes grayish white. Dorsum red, only head and tail tip black. Venter immaculate white.

\section{Discussion}

Apostolepis barrioi belongs to the A. dimidiata Group as defined by Ferrarezi et al. (2005) and Nogueira et al. (2012). The absence of dark coloration on the ventrals and subcaudals and the presence of two black dorsolateral stripes differentiate Apostolepis barrioi from other members of the group (Table 1). In A. albicollaris Lema, 2002, the belly is heavily pigmented, at least laterally (Nogueira et al. 2012), and in A. quirogai Giraudo and Scrocchi, 1998, the ventral pattern consists of dark ventrolateral stripes over the anterior third of the belly; the black coloration increases posteriorly (Giraudo and Scrocchi 1998). In these two species, the black coloration is restricted to some parts of the ventrals.

The resurrection of Apostolepis barrioi brings the number of species Apostolepis to 33 (Harvey 1999, Ferrarezi et al. 2005, Nogueira et al. 2012, Uetz et al. 2017), making it the most diverse genus of the tribe Elapomorphini. Further systematic study of the genus is recommended, especially for those species described the basis of only a few specimens.

\section{Acknowledgments}

We thank Elizabeth Paggini and Francisco Franco for permission to examine specimens of Apostolepis in their care, and Jean Paul Brouard and Fundación Para La Tierra and Nicolas Martinez (MNHNP) for photographs. Julian Faivovich and Santiago Nenda (MACN) provided data and photographs of the holotype of A. barrioi. We thank Pier Caccial and Ross MacCulloch, who reviewed earlier drafts of the manuscript, as well as Lía Romero and Jeffrey Thompson who reviewed our English usage. Jessica Fratani provided the Portuguese abstract. HC would like to thank Consejo Nacional de Ciencias y Tecnologías (CONACYT) for financial support through the Programa Nacional de Incentivo a Investigadores (PRONII).

\section{References}

Albuquerque, N. R. and T. Lema. 2012. Description of the second known specimen of Apostolepis intermedia (Serpentes, Colubridae, Xenodontinae). Zootaxa 3325: 53-58.

Amaral, A. 1921. Contribução para o conhecimento dos ofidios do Brazil. Parte I. Quatro noves espécies de serpentes brasileires. Memórias do Instituto Butantan 4: 1-68. 
Amaral, A. 1935. Estudos sobre ofídios nos neotrópicos XXXIII. Novas espécies de ofídios da Colômbia. Memórias do Instituto Butantan 9: 219-223.

Cope, E. D. 1887. Synopsis of the Batrachia and Reptilia obtained by H. H. Smith in the Province of Mato Grosso, Brazil. Proceedings of the American Philosophical Society 24: 44-60.

Curcio, F. F., P. M. S. Nunes, M. B. Harvey, and M. T. Rodrigues. 2011. Redescription of Apostolepis longicaudata (Serpentes: Xenodontinae) with comments on its hemipenial morphology and natural history. Herpetologica 67: 318-331.

Dinerstein, E., D. M. Olson, D. J. Graham, A. L. Webster, S. A. Primm, M. P. Bookbinder, and G. Ledec. 1995. Una Evaluación del Estado de Conservación de las Ecorregiones Terrestres de América Latina y el Caribe. Washington. Island Press. 174 pp.

Dowling, H. 1951. A proposed standard system of counting ventrals in snakes. British Journal of Herpetology 1: 97-99.

Duméril, A. M. C., G. Bibron, and A. H. A. Duméril. 1854. Erpétologie générale ou Histoire Naturelle complète des reptiles. Tome septième. Deuxième partie, comprenant l'historie des serpents venimeux. Paris. Librairie Encyclopédique de Roret: i-xii + 781-1536.

Entiauspe-Neto, O. M. and T. Lema. 2015. Apostolepis christineae Lema, 2002 (Serpentes: Xenodontinae: Elapomorphini): first record for Bolivia. Check List 11: $1-4$

Ferrarezi, H., F. E. Barbo, and C. E. Albuquerque. 2005. Phylogenetic relatio nship of a new species of Aposto lepis from Brazilian Cerrado with notes on the assimilis group (Serpentes: Colubridae: Xenodontinae: Elapomorphini). Papéis Avulsos de Zoología 45: 215-229.

Giraudo, A. R. 2002. Serpientes de la Selva Paranaense y del Chaco Húmedo. Buenos Aires. Literature of Latin America. 328 pp.

Giraudo, A. R. and G. J. Scrocchi. 1998. A new species of Apostolepis (Serpentes: Colubridae) and comments on the genus in Argentina. Herpetologica 54: 470-476.

Grazziotin, F. G., H. Zaher, R. W. Murphy, G. J. Scrocchi, M. A. Benavides, Y.-P. Zhang, and S. L. Bonatto. 2012. Molecular phylogeny of the New World Dipsadidae (Serpentes: Colubroidea): a reappraisal. Cladistics 88: 437-459.

Harvey, M. 1999. Revision of Bolivian Apostolepis (Squamata: Colubridae). Copeia 1999: 388-409.

Harvey, M. B., L. A. Gonzales, and G. J. Scrocchi. 2001. New species of Apostolepis (Squamata: Colubridae) from the Gran Chaco in Southern Bolivia. Copeia 2001: 501-207.

Lema, T. 1978. Novas espécies de opistoglifodontes do gênero Apostolepis Cope, 1861 do Paraguai (Ophidia: Colubridae: Colubrinae). Comunicações do Museu de Ciências PUCRGS 18/19: 27-49.

Lema, T. 1993. Polimorfismo em Apostolepis dimidiata (Jan, 1862), com a invalidação de Apostolepis villaricae Lema, 1978 e Apostolepis barrioi Lema, 1978 (Serpentes: Colubridae: Xenodontinae: Elapomorphini). Acta Biologica Leopoldensia 15: 35-52.

Lema, T. 1998. A redescription of the tropical Brazilian serpent Apostolepis nigrolineata (Peters 1869), (Colubridae: Elapomorphinae), synonymous with $A$. pymi Boulenger, 1903. Studies Neotropical Fauna and Environment 32: 193-199.

Lema, T. 2001. Fossorial snake genus Apostolepis from South America (Serpentes: Colubridae: Elapomorphinae). Cuadernos de Herpetología 15: 29-43.

Lema, T. 2002. Redescription of Apostolepis sanctaeritae Werner, 1924 (Serpentes, Colubridae) and a comparision with related species. Copeia 2002: 1147-1151.

Lema, T. 2003. Descrição de nova espécie de Apostolepis Cope, 1861 do Cerrado do Brasil, pertenecente ao grupo dimidiata (Serpentes, Elapomorphinae). Acta Biologica Leopoldensia 25: 123-131.

Lema, T. 2004a. Nova espécie de Apostolepis Cope do Estado de Rondônia, Brasil(Serpentes: Elapomorphinae). Comunicações do Museu de Ciências e Tecnologia PUCRGS, Série Zoologia 17: 81-89.

Lema, T. 2004b. New species of Apostolepis Cope, 1861 from Caatinga belonging to assimilis group (Serpentes, Elapomorphiniae). Comunicações do Museu de Ciências PUCRGS, Série Zoologia 17: 3-7.

Lema, T. 2016. Description of new species of Apostolepis (Serpentes: Dipsadidae: Xenodontinae: Elapomorphini) from Serra do Roncador, Central Brazil. Cuadernos de Pesquisa, Série Biologia 28: 1-12.

Lema, T. and M. Renner. 2004. New species of Apostolepis from Alto Tocantins, Brazil, with comments on the striped pattern species (Serpentes, Elapomorphinae). Biociências 12: 139-145.

Lema, T. and M. F. Renner. 2005. Contribution to the knowledge of Apostolepis cearensis Gomes, 1925 (Serpentes: Colubridae: Elapomorphinae), with comments on similar species from northeastern Brazil. Сотиnicações do Museu de Ciências Tecnologia PUCRGS, Série Zoologia 18: 129-140. 
Lema, T. and M. F. Renner. 2012. A new species of Apostolepis (Serpentes, Colubridae, Elapomorphini), belonging to assimilis group, found in Brazilian Cerrado. Ciência em Movimento 27: 71-76.

Lema, T. and N. R. Albuquerque. 2010. The identity of Apostolepis pymi and placement of A. quinquelineata in the synonymy of A. nigrolineata (Serpentes, Xenodontinae). Biota Neotropica 10: 343-346.

Lema, T. and C. Hofstadler-Deiques. 2010. Description of a new genus for allocation of Elapomorphus lepidus and the status of Elapomorphus wuchereri (Serpentes: Dipsadidae: Xenodontinae: Elapomorphini). Neotropical Biology and Conservation 5: 113-119.

Loebmann, D. and T. Lema. 2012. New data on the distribution of the rare and poorly known Apostolepis goiasensis Prad, 1943 (Serpentes, Xenodontinae, Elapomorphini) with remarks on morphology and colouration. Herpetology Notes 5: 523-525.

Nogueira, C., F. E. Barbo, and H. Ferrarezzi. 2012. Redescription of Apostolepis albicollaris Lema, 2002, with a key for the species groups of the genus Apostolepis (Ser pentes: Dipsadidae: Elapo morphini). South American Journal of Herpetology 7: 213-225.

Peters, J. and B. Orejas-Miranda. 1972. The taxonomic validity of Apostolepis tenius Ruthven and Apostolepis vittata (Cope) (Serpentes: Colubridae). Copeia 1972: 588-590.

Peracca, M. 1904. Viaggio del Dr. A. Borelli nel Matto Grosso brasiliano e nel Paraguay, 1899. IX. Rettili ed amfibii, Bolletino del Musei di Zoologia ed Anatomia Comparada 19: 1-15.
Pyron, R. A., F. T. Burbrink, and J. J. Wiens. 2013.A phylogeny and revised classification of Squamata, including 4161 species of lizards and snakes. BMC Evolutionary Biology 13: 1-53.

Rodrigues, M. T. 1993. Herpetofauna das dunas interiores do Rio São Francisco: Bahia: Brasil. V. Duas novas espécies de Apostolepis (Ophidia, Colubridae). Memórias do Instituto Butantan 54: 53-59.

Ruthven, A. G. 1927. Description of an apparently new species of Apostolepis from Bolivia. Occasional Papers of the Museum of Zoology, University of Michigan 188: $1-2$.

Sabaj-Pérez, M. H. 2016. Standard symbolic codes for institutions resource collections in herpetology and ichthyology: an online reference. Version 6.5. Electronic Database accessible at http://www.asih.org, American Society of Ichthyologists and Herpetologists, Washington, USA.

Uetz, P., P. Freed, and J. Hosêk (eds.). 2017. The Reptile Database. Electronic Database accessible at http://www. reptile-database.org. Captured on 18 July of 2017.

Zaher, H. 1999. Hemipenial morphology of the South American Xenodontinae snakes, with a proposal for a monophyletic Xenodontinae and a reappraisal of colubroid hemipenis. Bulletin of the American Museum of Natural History 240: 1-168.

Zaher, H., F. G. Grazziotin, J. E. Cadle, R. W. Murphy, J. C. Moura-Leite, and S. L. Bonatto. 2009. Molecular phylogeny of advanced snakes (Serpentes, Caenophidia) with an emphasis on South American Xenodontines: a revised classification and descriptions of new taxa. Papéis Avulsos de Zoologia 49: 115-153.

Editor: Ross D. MacCulloch 
Cabral et al.

Appendix I. Specimens examined.

Apostolepis barrioi: whithout locality: IBSP 204, IBSP 214, IBSP 231, IBSP 280, IBSP 328, IBSP 414, IBSP 422, IBSP 434, IBSP 443, IBSP 445, IBSP 448, IBSP 474, IBSP 483, IBSP 529, IBSP 583. BRAZIL: IBSP 3760. Mato Gross Do Sul: Aquidauana, LPH 130, earlier CHAFB 902; Dourados, IBSP 40425; Rio Brilhante, IBSP 42721. MinAs GeraIS: Guia Lopes da Laguna LPH 129 earlier CHAFB.902; Uberlândia UFU 757. São PAulo: Araraquara: IBSP 23363, Severino Farm IBSP 46535; Atalaia IBSP 8521; Brotas IBSP 32759, Elba Farm IBSP 42264; Caetetuba IBSP 4509; Jardim dos Lagos IBSP 12347; Faveiro: Rio das Pedras IBSP 355, IBSP 539, IBSP 540; Paraisópolis IBSP 20359; Ribeirão Preto IBSP 117, Tambaú IBSP 4817; Santa Adélia: Terra Roxa IBSP 5809; Toriba IBSP 10274. PARAGUAY: Amambay: Cerro Cora National Park, one km N from Park Administration Building MHNP 5162, formerly USNM 97053. CONCEPCIÓN: Cororo, Ypane River MACN 49402 formerly CHINM.3309, holotype of A. barrioi. SAN PEdro: Private Reserve Laguna Blanca MNHNP 11548, Colonia Primavera BMNH.1955.1.6.1, BMNH.1960.1.3.3.

Apostolepis dimidiata: BRAZIL: without locality: NMW 21992. Mato Grosso do Sul: Maracaju AMNH.62192; Miranda MLP 577, holotype A. ventrimaculatus IBSP 5626; Salobra, km 122 railroad NW MNRJ 763. Rio DE JANEIRO: Joaquin Leite IBSP 8515. São PAulo: Água Vermelha IBSP 7747; Americana IBSP 42589, IBSP 47513; Anísio de Morais IBSP 1187; Araçatuba IBSP 7524; Araçoiaba da Serra IBSP 29232; Araraquara IBSP 19970; Distrito Américo, Boa Esperança Farm IBSP 46535, IBSP 48992; Assis IP 1901, IBSP 1905, IBSP 23226, IBSP 30861, IBSP 44471, IBSP 49205, IBSP 49688; Atalaia IBSP 9108; Atibaia IBSP 6084, IBSP 40402; Avaré IBSP 22895; Batoví IBSP 19131; Bernardino de Campos IBSP 27371; Botucatu IBSP 5669, AMNH.72425; Brotas IBSP 32759, IBSP 40520; Elba Farm IBS 42264; Caetetuba IBSP 5905; Caiuá IBSP 7029; Campinas IBSP 44477; Capivari IBSP 9353; Casa Branca IBSP 1393; Cerqueira César IBSP 7880; Conchas IBSP 118; Corumbataí IBSP 425; Cruzália IBSP 27671; Echaporã (= Cocaramurú) IBSP 31237; Faveiro, Rio das Pedras IBSP 119, IBSP 2547; Ferdinando Laboriau IBSP 5803; Fernão Dias IBSP 8024; Gália IBSP 40733; Garça IBSP 27568; Guaianás IBSP 8121; Guarani IBSP 1786; Guarantã IBSP 10357; Guararapes IBSP 5593; Herculândia IBSP 34117; Ibarra IBSP 8021; Indaiatuba IBSP 45198; Ipanema IBSP 4566, ZIB 5822 holotype of Elapomorphus erythronotus, lost; Itaguá IBSP 4546, IBSP 5066, IBSP 5873; Itapetininga IBSP 9507, IBSP 29640, IBSP 41354; Itápolis IBSP 6041; Itararé IBSP 9349, IBSP 49007; Itatinga IBSP 3294; Itobí IBSP 7335, IBSP 7603; Jaú IBSP 42727; Lauro Müller IBSP 5537; Lençóis IBSP 1362; Limeira IBSP 812, IBSP 47371; Lins IBSP 43894; Lucélia IBSP 31631; Mairiporã IBSP 42527; Maracanã IBSP 10066; Matão IBSP 4747, IBSP 5773; Miguel Calmon MCZ 2766o, USNM 76369; Motuca IBSP 16665; Nova Odessa IBSP 3319; Novo Horizonte IBSP 1631; Olímpia IBSP 9340, IBSP 40413; Palmas IBSP 7736; Palmital IBSP 27688; Paraiso IBSP 15657; Paranapuã IBSP 16733, IBSP 32483; Paula Souza IBSP 3063; Pederneiras IBSP 9825, IBSP 9829; PeixotoGomide IBSP 12745; Penápolis IBSP 8819, IBSP 31049; Piracicaba IBSP 42123, IBSP 42128; Pirassununga: Emas MP 100, MP 102, MP 103, MP 104, MP 1891; IBSP 8986, IBSP 9479; Pirajú IBSP 7415, IBSP 9957, IBSP 16734; Platina IBSP 27842; Pompéia IBSP 46141; Presidente Wenceslau IBSP 34279; Prudentópolis IBSP 5733; Rancharia IBSP 32396, AMNH 102242; Ribeirão Preto IBSP 25604; Rinópolis IBSP 16644; Rubião Júnior IBSP 7271; Santa Ernestina IBSP 923, IBSP 1176; Santa Lúcia: Sta. Izabel Farm IBSP 44543; Santa Rosa do Viterbo IBSP 19059; São Paulo ZSM 197-10a, syntype A. nigriceps, lost; NMW 21990; Sapezal IBSP 7690; Tambaú IBSP 32112; Toriba IBSP 10297, IBSP 10298; Torrinha IBSP 32447; Tupã: Universo IBSP 17175; Valinhos IBSP 4494; Valparaiso IBSP 14524, IBSP 31901; Varnhagen, near Ipanema Farm FMNH 1645; Vila Costner IBSP 3095; Votuporanga IBSP 41048. PARANÁ: IBSP 5325, NMW 13807; Campo Mourão IBSP 49624; Centenário do Sul MHNCI 3086. PARAGUAY: GuAIRA: Ybytyruzu National Park CZ 741.

Apostolepis aff. dimidiata: ARGENTINA: bordering Uruguay River IBSP 7781. CoRRIENTES: UNNEC 147, $189,249$. Misiones: CUNAM 002, 003, 004; UNNEC 183; CUNAM 237, 850.

Apostolepis spp.: Without locality: ZSM 197-10b, syntype of A. nigriceps; IBSP 727, IBSP 3066, IBSP3333, IBSP 3760, IBSP 3801, IBSP 7836, IBSP 9738, IBSP 9739, IBSP 9759, IBSP 20539, IBSP 48102, IBSP 53547, IBSP 53110. 